\title{
Stability of delayed ratio-dependent predator-prey system
}

\author{
Sándor Kovács
}




\title{
STABILITY OF A DELAYED RATIO-DEPENDENT PREDATOR-PREY SYSTEM
}

\author{
SÁNDOR KOVÁCS
}

[Received: April 4, 2003]

\begin{abstract}
A ratio-dependent predator-prey model is considered in which the predator growth rate depends on past quantities of the prey. Conditions for stability of an equilibrium and its bifurcation are established when special parameters are taken into account.

Mathematics Subject Classification: Primary 92A15; Secondary 34C15, 34K15
\end{abstract}

Keywords: Ratio-dependent, delay, Poincaré-Andronov-Hopf bifurcation

\section{InTRODUCTION}

In $[15,16,24]$, a possible generalisation of the traditional Volterra predator-prey system is considered,

$$
\begin{aligned}
& \dot{S}_{1}=S_{1} \cdot \alpha\left(S_{1}\right)-S_{2} \cdot V\left(S_{1}\right), \\
& \dot{S}_{2}=S_{2} \cdot K\left(S_{1}\right)
\end{aligned}
$$

where the dot means differentiation with respect to time $t, S_{1}(t)$ and $S_{2}(t)$ are the quantities (or densities) of preys and predators, respectively. Here,

- $\alpha$ is smooth with $\alpha^{\prime}\left(S_{1}\right)<0, S_{1} \geq 0$ and $\alpha(0)>0>\lim _{+\infty} \alpha$;

- $K$ and $V$ are nonnegative and increasing, and $K(0)=0=V(0)$.

If we replace the argument of $K$ and $V$ by the ratio of $S_{1}$ and $S_{2}$, then we arrive at a ratio-dependent predator-prey system, which is capable of producing richer and more reasonable or acceptable dynamics. The substantial difference from the classical Kolmogorov model $[15,16]$ is due to the following two facts related to the system:

(i) Equilibrium abundances are positively correlated along a gradient of enrichment [1];

(ii) These models do not produce the so-called paradox of enrichment (see [11, pp. 490, 502] and [17, p. 391]), more exactly, it either completely disappears or enrichment is related to stability in a more complicated way [21]. 
A simple ratio-dependent type mathematical model of two-species interaction was first presented in $[10,19,21]$.

Let us consider a model of ratio-dependent type which has the form

$$
\begin{aligned}
& \dot{S}_{1}=f_{1}\left(S_{1}, S_{2}\right), \\
& \dot{S}_{2}=f_{2}\left(S_{1}, S_{2}\right)
\end{aligned}
$$

with the initial conditions $S_{i}(0)>0(i \in\{1,2\})$, where

$$
f_{1}\left(S_{1}, S_{2}\right):= \begin{cases}\alpha S_{1}\left(1-\frac{S_{1}}{K}\right)-\frac{\beta S_{1} S_{2}}{\varepsilon S_{2}+S_{1}} & \text { if } S_{1}^{2}+S_{2}^{2}>0 \\ 0 & \text { if } S_{1}=S_{2}=0\end{cases}
$$

and

$$
f_{2}\left(S_{1}, S_{2}\right):= \begin{cases}-\gamma S_{2}+\frac{\delta S_{1} S_{2}}{\varepsilon S_{2}+S_{1}} & \text { if } S_{1}^{2}+S_{2}^{2}>0 \\ 0 & \text { if } S_{1}=S_{2}=0 .\end{cases}
$$

Here, $\alpha>0$ is the intrinsic growth rate of $S_{1}$ in the absence of the $S_{2}$-population and without environmental limitation. In the absence of the $S_{2}$-population, the $S_{1^{-}}$ population grows logistically to the carrying capacity $K>0$; the functional response of the $S_{2}$-population is of Michaelis-Menten-Holling type with satiation coefficient or conversion rate $\delta>0$. The specific mortality

$$
E\left(S_{2}\right)=-\gamma S_{2}
$$

of the $S_{2}$-population in the absence of the $S_{1}$-population depends on the quantity of $S_{2} . \gamma>0$ is the death rate of the $S_{2}$-population.

If we take polar coordinates $x=r \cos \varphi, y=r \sin \varphi$, then a routine calculation shows that $f_{i} \in C^{0}\left(\mathbb{R}_{+}^{2}, \mathbb{R}\right)$, however, it is easy to see that $f_{i} \in C^{1}\left(\mathbb{R}_{+}^{2}, \mathbb{R}\right)(i \in\{1,2\})$, therefore solution of (1.2) with positive initial condition exists and is unique.

Let $S_{1} \cdot M_{1}\left(S_{1}, S_{2}\right):=f_{1}\left(S_{1}, S_{2}\right)$ and $S_{2} \cdot M_{2}\left(S_{1}, S_{2}\right):=f_{2}\left(S_{1}, S_{2}\right)$, then the system (1.2) is written in the Kolmogorov form of

$$
\dot{\mathbf{S}}=\left(\begin{array}{l}
S_{1} \cdot M_{1}(\mathbf{S}) \\
S_{2} \cdot M_{2}(\mathbf{S})
\end{array}\right)
$$

(see [19]), which is useful since one can check the following two properties of this system:

- $M_{1}$ and $M_{2}$ are smooth functions, therefore the positive quadrant of the phase space $\left[S_{1}, S_{2}\right]$ is an invariant region (see [22], pp. 198-203 and 230231);

- $\frac{\partial M_{1}}{\partial S_{2}}\left(S_{1}, S_{2}\right)=-\frac{\beta}{\varepsilon+S_{1}}<0$ and $\frac{\partial M_{2}}{\partial S_{1}}\left(S_{1}, S_{2}\right)=-\frac{\delta \varepsilon}{\left(\varepsilon+S_{1}\right)^{2}}>0\left(S_{1}, S_{2}>0\right)$, i. e., in fact the system is a predator-prey system with prey $S_{1}$ and predator $S_{2}$.

The ratio-dependent predator-prey model (1.2) has been studied by several authors recently, and very rich dynamics has been observed. In [10] and later in [2], the authors restricted their analysis to parameter values that ensure that the origin as equilibrium behaves as a saddle point and they established conditions for persistence 
of the model, and showed the existence of eight qualitatively different types of system behaviours realized for various parameter values. In [13] and later in [26] the authors studied the analytical behaviour at the origin and showed that this equilibrium can be either a saddle point or an attractor of certain trajectories, and shown that the origin is indeed a critical point of higher order. In [17] the global behaviour of solutions was investigated and it was showed that if the positive equilibrium is locally asymptotically stable, then the system does not have any nontrivial positive periodic solutions.

Now we are going to show that with respect to certain circumstances this model can have a periodic solution. This happens by incorporating a delay effect into the systems. For the justification and biological relevance of the delay in ratio-dependent systems see [6]. In [14] the most famous delay in the predator-equation was introduced, i. e., $K\left(\frac{S_{1}(t)}{S_{2}(t)}\right)$ was replaced by $K\left(\frac{S_{1}(t-\tau)}{S_{2}(t-\tau)}\right)$. Starting from the evidence that in such a system the present growth rate of a predator depends not only on the present quantity of food but also on past quantities, we will introduce an infinite distributed delay into the second equation of system (1.2) for prey density, i. e., we replace $S_{1}$ in the second equation by

$$
R(t):=\int_{-\infty}^{t} S_{1}(\tau) \rho(t-\tau) d \tau, \quad t \in[0,+\infty),
$$

where $\rho: \mathbb{R}_{0}^{+} \rightarrow \mathbb{R}_{0}^{+}$is the so-called density function satisfying the relation

$$
\int_{0}^{+\infty} \rho(s) d s=1
$$

Due to $[5,4,7,8,18,25]$, we assume that the influence of the past is fading away exponentially, i. e., $\rho(t):=a \cdot \exp (-a t)(a \in(0,+\infty))$ and $a \int_{-\infty}^{t} \exp (-a(t-\tau)) d \tau=$ $a \int_{0}^{+\infty} \exp (-a s) d s=1$ hold. The smaller $a$ is, the longer the time interval is in the past in which the values of $S_{1}$ are taken into account, i. e., $1 / a$ is the "measure of the influence of the past".

Clearly, if $\gamma<\delta<\frac{\gamma \beta}{\beta-\varepsilon \alpha}$ for $\gamma>\varepsilon \alpha$ or $\delta>\gamma$ for $\gamma \leq \varepsilon \alpha$, then (1.2) has three equilibria, two on the boundary of the positive quadrant: $(0,0)^{T}$, whose local stability cannot be directly studied (the system cannot be linearised), $(K, 0)^{T}$ that is unstable and a unique equilibrium with positive coordinates: $\left(\overline{S_{1}}, \overline{S_{2}}\right)^{2}:=\frac{K}{\alpha \varepsilon \delta}(\beta(\gamma-\delta)+$ $\delta \varepsilon \alpha)\left(1, \frac{\delta-\gamma}{\gamma \varepsilon}\right)^{T}$ which may or may not be stable. To study the local stability of equilibrium $(0,0)^{T}$ there are several ways, e. g. via introducing the new variable $S:=S_{1} / S_{2}$ or making a time scale change $d t=:\left(\varepsilon S_{2}+S_{1}\right) d \tau$ (cf. [17] and [26]). First we give conditions for the asymptotic stability of the equilibrium of the delayed system and show that under some conditions the increase of the delay $\frac{1}{a}$ destabilises the originally stable equilibrium by a Poincaré-Andronov-Hopf bifurcation (see [9]). While the system without delay has no periodic solutions apart from the trivial one, this one has. 


\section{The SYSTEM WITH DELAY}

We consider the model of predator-prey interaction with time delay which is given by

$$
\begin{aligned}
& \dot{S}_{1}=\alpha S_{1}\left(1-\frac{S_{1}}{K}\right)-\frac{\beta S_{1} S_{2}}{\varepsilon S_{2}+S_{1}} \\
& \dot{S}_{1}=-\gamma S_{2}+\frac{\delta R S_{2}}{\varepsilon S_{2}+R}
\end{aligned}
$$

where $R$ is given by (1.5). Since

$$
\dot{R}(t)=a\left(S_{1}(t)-R(t)\right) \quad(t \in[0,+\infty)),
$$

we see that (2.1) is equivalent in its qualitative dynamical behaviour to the three dimensional system of ordinary differential equations

$$
\begin{aligned}
\dot{S_{1}} & =\alpha S_{1}\left(1-\frac{S_{1}}{K}\right)-\frac{\beta S_{1} S_{2}}{\varepsilon S_{2}+S_{1}} \\
\dot{S_{1}} & =-\gamma S_{2}+\frac{\delta R S_{2}}{\varepsilon S_{2}+R} \\
\dot{R} & =a\left(S_{1}-R\right)
\end{aligned}
$$

on $[0, \infty)$ in the following sense (see also [7]). If $\left(S_{1}, S_{2}\right):[0,+\infty) \rightarrow \mathbb{R}^{2}$ is the solution of (2.1) corresponding to the continuous and bounded initial function $\tilde{S_{1}}$ : $(-\infty, 0] \rightarrow \mathbb{R}$ and the initial value $S_{2}^{0}:=S_{2}(0)$ (i. e., $\left.S_{1}(t):=\tilde{S_{1}}(t)(t<0)\right)$, then $\left(S_{1}, S_{2}, R\right):[0,+\infty) \rightarrow \mathbb{R}^{3}$ is the solution of (2.2) satisfying the initial conditions $S_{1}(0)=\tilde{S_{1}}(0), S_{2}(0)=S_{2}^{0}$ and $R(0)=R^{0}:=a \int_{-\infty}^{0} \tilde{S_{1}}(\tau) \exp (a \tau) d \tau$ and vice versa. (Clearly, if the initial values $S_{1}(0), S_{2}^{0}$ and $R^{0}$ related to system (2.2) are prescribed then, the function $\tilde{S_{1}}$ is not uniquely determined.) There are seven parameters in (2.2), thus if we introduce new variables and time with substitutions such as

$$
S_{1}=: K u, \quad S_{2}=: \frac{K}{\varepsilon} v, \quad R=: K w, \quad t=: \alpha \tau,
$$

then (2.2) takes the following simpler, dimensionless form

$$
\begin{aligned}
u^{\prime} & =u(u-1)-\frac{b u v}{v+u} \\
v^{\prime} & =-c d v+\frac{c v w}{v+w} \\
w^{\prime} & =\mu(u-w)
\end{aligned}
$$

where $b:=\frac{\beta}{\alpha \varepsilon}, c:=\frac{\delta}{\alpha}, d:=\frac{\gamma}{\delta}$ and $\mu:=\frac{a}{\alpha}$, and where the prime, this time, denotes differentiation with respect to the variable $\tau$. With these notations, if $0<d<1<$ $b<\frac{1}{1-d}$ for $b>1$ or if $0<d<1$ for $b \leq 1$, then system (2.3) has the following equilibria: firstly, the origin, whose local stability cannot be directly investigated and is of no interest, secondly, the points $(1,0,1)^{T}$ and the unique positive $(\bar{u}, \bar{v}, \bar{w})^{T}:=$ 
$\left(b(d-1)+1, \frac{1-d}{d}(b(d-1)+1), b(d-1)+1\right)^{T}$, which represent the extinction state of predator and the coexistence state of predator and prey, respectively.

In order to check the stability of the last two equilibria we linearise system (2.3) at these points. The coefficient matrix is

$$
A(u, v, w):=\left[\begin{array}{ccc}
1-2 u-\frac{b v^{2}}{(v+u)^{2}} & -\frac{b u^{2}}{(v+u)^{2}} & 0 \\
0 & \frac{c w^{2}}{(v+w)^{2}}-c d & \frac{c v^{2}}{(v+w)^{2}} \\
\mu & 0 & -\mu
\end{array}\right] .
$$

In particular,

$$
A(1,0,1)=\left[\begin{array}{ccc}
-1 & -b & 0 \\
0 & c(1-d) & 0 \\
\mu & 0 & -\mu
\end{array}\right], \quad A(\bar{u}, \bar{v}, \bar{w})=\left[\begin{array}{ccc}
e k-1 & -b d^{2} & 0 \\
0 & c d k & c k^{2} \\
\mu & 0 & -\mu
\end{array}\right],
$$

where $e:=b(1+d), k:=1-d$, and the characteristic polynomials take the form

$$
p_{101}(\lambda):=\lambda^{3}+(1+\mu-c(1-d)) \lambda^{2}+(\mu-\mu c(1-d)-c(1-d)) \lambda+\mu c(1-d)
$$

and

$p_{\overline{u v w}}(\lambda):=\lambda^{3}+(\mu+c d k-e k+1) \lambda^{2}+(c d k(\mu+1)-e k(\mu-c d k)+\mu) \lambda+\mu c d k(e k+1+b d k)$.

Applying the Routh-Hurwitz criterion, we conclude that they are stable polynomials if and only if the following inequalities hold:

$$
\begin{gathered}
1+\mu>c(1-d), \\
\mu(1-c(1-d))>c(1-d), \\
\mu c(1-d)>0,
\end{gathered}
$$

$$
P_{101}(\mu):=(1-c(1-d)) \mu^{2}+\left(1-4 c(1-d)+c^{2}(1-d)\right) \mu+c(1-d)(c-1)>0
$$

and

$$
\begin{gathered}
\mu+c d k+1>e k \\
\mu(c d k-e k+1)>-c d k(1-e k) \\
\mu c d k(e k+1+b d k)>0,
\end{gathered}
$$

and

$$
\begin{aligned}
P_{\overline{u v w}}(\mu):=(c d k-e k+1) \mu^{2}+(c d k(c d k+2-2 e k-b d k) \\
+e k(e k-2)+1)) \mu+c d k(1+c d k(1+e k)-e k)>0 .
\end{aligned}
$$

If we assume that

$$
1-e k>0
$$

i. e.,

$$
\frac{1}{1-d}>b(1+d)
$$


then (2.5a) and (2.5b) hold. Clearly, (2.5c) holds automatically, thus (2.5d) and (2.6a) (resp. (2.6b)) atogether form a sufficient condition of asymptotic stability of the equilibrium $(\bar{u}, \bar{v}, \bar{w})^{T}$.

In view of (2.6a), the following three cases can be distinguished.

2.1. Case $e \boldsymbol{k}<1$. In this case the inequalities $(2.5 \mathrm{a}-\mathrm{c})$ hold true. If

$$
c d k(c d k+2-2 e k-b d k)+e k(e k-2)+1 \geq 0,
$$

then (2.5d) holds for all $\mu$ and $(\bar{u}, \bar{v}, \bar{w})^{T}$ is asymptotically stable. If (2.7) does not hold, then since the constant term of the quadratic polynomial $P_{\overline{u v w}}$ is positive, this polynomial has either no real roots or has two roots of the same sign. If $P_{\overline{u v w}}$ has no real roots or has two negative roots, then (2.7) holds again for all $\mu$ and the equilibrium $(\bar{u}, \bar{v}, \bar{w})^{T}$ is asymptotically stable. If $P_{\overline{u v w}}$ has two positive roots, $0<\mu_{1}<\mu_{0}$, say, then the equilibrium $(\bar{u}, \bar{v}, \bar{w})^{T}$ is asymptotically stable for large values of $\mu$, i. e., for small delays. At $\mu_{0}$, the characteristic polynomial $p_{\overline{u v w}}$ has the form

$$
p_{\overline{u v w}}(\lambda) \equiv\left(\lambda^{2}+c d k\left(\mu_{0}+1\right)-e k\left(\mu_{0}-c d k\right)+\mu_{0}\right)\left(\lambda+\mu_{0}+c d k-e k+1\right),
$$

and its roots are

$$
\lambda_{0}\left(\mu_{0}\right)=-\mu_{0}-c d k+e k-1<0 \quad \text { and } \quad \lambda_{1,2}\left(\mu_{0}\right)= \pm i \omega
$$

where

$$
\omega:=\sqrt{c d k\left(\mu_{0}+1\right)-e k\left(\mu_{0}-c d k\right)+\mu_{0}} .
$$

A routine calculation shows that

$$
\begin{aligned}
& \frac{d \mathfrak{R}\left(\lambda_{1}\left(\mu_{0}\right)\right)}{d \mu} \\
& \quad=-\frac{\left(c d k\left(\mu_{0}+1\right)-e k\left(\mu_{0}-c d k\right)+\mu_{0}-3 \omega^{2}\right)\left(c d k(e k+1)+b d k-\omega^{2}\right)}{\left(c d k\left(\mu_{0}+1\right)-e k\left(\mu_{0}-c d k\right)+\mu_{0}-3 \omega^{2}\right)^{2}+4 \omega^{2}\left(\mu_{0}+c d k-e k+1\right)^{2}} .
\end{aligned}
$$

Example. Let $b=2.0000, d=0.1000, c=0.1000$, then the expression on the left hand side in (2.7) is negative, $\mu_{0}=0.4319$ and $\omega=0.5970$, furthermore,

$$
\frac{d \mathfrak{R}\left(\lambda_{1}\left(\mu_{0}\right)\right)}{d \mu}=-\frac{0.2187}{2.5511}<0,
$$

therefore using $\mu$ as bifurcation parameter, the equilibrium $(\bar{u}, \bar{v}, \bar{w})^{T}$ looses its stability by a Poincaré-Andronov-Hopf bifurcation when $\mu$ is decreased below $\mu_{0}$, i. e., the delay is increased, while the other equilibrium becomes asymptotically stable. 
2.2. Case $\boldsymbol{e} \boldsymbol{k}=1$. In this case the inequalities $(2.5 \mathrm{a}-\mathrm{c})$ hold true, $(2.5 \mathrm{~d})$ is equivalent to

$$
P_{\overline{u v w}}(\mu):=\mu^{2}+d k(c-b) \mu+2 c d k>0 .
$$

If $c-d \geq 0$, then (2.8) holds for all $\mu$ and $(\bar{u}, \bar{v}, \bar{w})^{T}$ is asymptotically stable. If $c<d$, then since the constant term of the quadratic polynomial $P_{\overline{u v w}}$ is positive, this polynomial has either no real roots or has two roots of the same sign. If $P_{\overline{u v w}}$ has no real roots or has two negative roots, then (2.8) holds again for all $\mu$ and the equilibrium $(\bar{u}, \bar{v}, \bar{w})^{T}$ is asymptotically stable. If $P_{\overline{u v w}}$ has two positive roots, $0<$ $\mu_{1}<\mu_{0}$, say, then the equilibrium $(\bar{u}, \bar{v}, \bar{w})^{T}$ is asymptotically stable for large values of $\mu$, i. e., for small delays. At $\mu_{0}$ the characteristic polynomial $p_{\overline{u v w}}$ has the form

$$
p_{\overline{u v w}}(\lambda) \equiv\left(\lambda^{2}+c d k\left(\mu_{0}+1\right)-\left(\mu_{0}-c d k\right)+\mu_{0}\right) \times\left(\lambda+\mu_{0}+c d k\right),
$$

whose roots are

$$
\lambda_{0}\left(\mu_{0}\right)=-\mu_{0}-c d k<0, \quad \text { and } \quad \lambda_{1,2}\left(\mu_{0}\right)= \pm i \omega,
$$

where $\omega:=\sqrt{2 c d k\left(\mu_{0}+1\right)}$. A routine calculation shows that

$$
\begin{aligned}
& \frac{d \mathfrak{R}\left(\lambda_{1}\left(\mu_{0}\right)\right)}{d \mu} \\
& \quad=-\frac{\omega^{2}\left(3 \omega^{2}+c d k\left(4 \mu_{0}-2 c d k\left(1+\mu_{0}\right)-2-3 c d^{2} k^{2}\right)\right)+2 c^{2} d^{2} k^{2}(2+b d k)}{\left(2 c d k\left(\mu_{0}+1\right)-3 \omega^{2}\right)^{2}+4 \omega^{2}\left(\mu_{0}+c d k\right)^{2}} .
\end{aligned}
$$

Example. Let $b=0.999900000, c=0.000000001$, then $d=0.000050003$ and (2.8) is negative, $\mu_{0}=0.00054791$ and $\omega=0.999999998$, furthermore,

$$
\frac{d \mathfrak{R}\left(\lambda_{1}\left(\mu_{0}\right)\right)}{d \mu}=-0.031314697<0
$$

therefore in this case the equilibrium $(\bar{u}, \bar{v}, \bar{w})^{T}$ looses its stability if $\mu$ is decreased below $\mu_{0}$. This loss of stability occurs again by a Poincaré-Andronov-Hopf bifurcation, while the other equilibrium becomes asymptotically stable.

2.3. Case $\boldsymbol{e} \boldsymbol{k}>\mathbf{1}$. In this case (2.5a-b) are not satisfied automatically. If we assumed that $c d>e$, then (2.5a) would hold but we have no guarantee that (2.5b) will hold, so it is not sure even for the value $\mu\left(\mu<\mu_{0}\right)$ that the polynomial $p_{\overline{u v w}}(\lambda)$ is stable.

\section{REFERENCES}

[1] Ariditi, R. and GrinzbuRg, L. R.: Coupling in predator-prey dynamics: ratio-dependence, J. Theoretical Biology, 139 (1989), 311-326.

[2] Berezovskaya, F., Karev, G. and Arditi, R.: Parametric analysis of the ratio-dependent predatorprey model, J. Math. Biol., 43 (2001), 221-246.

[3] Berryman, A. A.: The origins and evolution of predator-prey theory, Ecology, 73 (1992), 15301535.

[4] CAVANI, M. and Farkas, M.: Bifurcations in a predator-prey model with memory and diffusion. I: Andronov-Hopf bifurcation, Acta Math. Hungar., 63(3) (1994), 213-229. 
[5] Cushing, J. M.: Integrodifferential Equations and Delay Models in Population Dynamics, Lecture Notes in Biomathematics, 20, Berlin: Springer Verlag, 1977.

[6] DeAngelis, D. L., Goldstein, R. A. and O'Neill, R. V.: A model for trophic interaction, Ecology, 56 (1975), 881-892.

[7] Farkas, M.: Stable oscillations in a predator-prey model with time lag, J. Math. Anal. Appl., 102 (1984), 175-188.

[8] Farkas, A., Farkas, M., and Szabó, G.: Multiparameter bifurcation diagrams in predator-prey models with time lag, J. Math. Biol., 26 (1988), 93-103.

[9] Farkas, M.: Periodic Motions, Berlin, Heidelberg and New York: Springer Verlag, 1994.

[10] Freedman, H. I. and Mathsen, R. M.: Persistence in predator-prey systems with ratio-dependent predator influence, Bull. Math. Biol., 55 (1993), 817-827.

[11] Hsu, S. B., Hwang, T. W., and Kunng, Y.: Rich dynamics of a ratio-dependent one-prey twopredators model, J. Math. Biol., 42 (2001), 489-506.

[12] Hsu, S. B., Hwang, T. W., and Kunng, Y.: Rich dynamics of a ratio-dependent one-prey twopredators model, J. Math. Biol., 43 (2001), 377-396.

[13] Jost, C., Arino, O., And Arditi, R.: About deterministic extinction in ratio-dependent predatorprey models, Bull. Math. Biol., 61 (1999), 19-32.

[14] Kesh, D., Mukherjee, D., Sarkar, A. K., and Roy, A. B.: Ratio dependent predation: A bifurcation analysis, Korean J. Comput \& Appl. Math., 5 (1998), No. 2, 295-305.

[15] Kolmogorov, A. N.: Sulla teoria di Volterra della lotta per l'esistence, G. Inst. Ital. Attuari, Mir, 7, 1 (1936), pp. 74-80.

[16] Kolmogorov, A. N.: Qualitative analysis of mathematical models of populations, Problems of cybernetics, Nauka, Moscow, 25 (1972), pp. 100-106.

[17] Kuna, Y. AND Beretta, E.: Global qualitative analysis of a ratio-dependent predator-prey system, J. Math. Biol., 36 (1998), 389-406.

[18] MacDonald, N.: Time delay in predator-prey models, II. Bifurcation theory, Math. Biosci., 33 (1977), 227-234.

[19] May, R.: Stability and Complexity in Model Ecosystems, Monographs in Population Biology, Princeton University Press, Princeton, 1973.

[20] Maynard Smith, J.: Models in Population Ecology, Cambridge Univ. Press, 1974.

[21] Rosenzweig, M. L.: Paradox of enrichment: destabilization of exploitation ecosystems in ecological time, Science, 171 (1969), 385-387.

[22] Smoller, J.: Shock Waves and Reaction-Diffusion Equations, Springer (Berlin), 1983.

[23] STÉpÁn, G.: Great delay in a predator-prey model, Nonlin. Anal. TMA., 10 (1986), 913-929.

[24] Svirezhev, Yu. M. And Logofet, D. O.: Stability of Biological Communities, Moscow, Mir, 1983.

[25] Szabó, G.: A remark on M. Farkas: Stable oscillations in a predator-prey model with time-lag, J. Math. Anal. Appl., 128 (1987), 205-206.

[26] Xiao, D. and Ruan, S.: Global dynamics of a ratio-dependent predator-prey system, J. Math. Biol., 43 (2001), 268-290.

\section{Author's Address}

\section{Sándor Kovács:}

Department of Numerical Analysis, Eötvös L. University, P. O. Box 32, H-1518 Budapest, HunGARY

E-mail address: alex@ludens.elte.hu 